\title{
DESEMPENHO COGNITIVO-LINGUÍSTICO E EM LEITURA DE ESCOLARES COM TRANSTORNO DE DÉFICIT DE ATENÇÃO E HIPERATIVIDADE
}

\author{
PERFORMANCE COGNITIVE-LINGUISTIC AND \\ READING OF STUDENTS WITH ATTENTION DEFICIT \\ AND HYPERACTIVITY DISORDER
}

\author{
Cláudia da Silva ${ }^{1}$, Vera Lúcia Orlandi Cunha ${ }^{2}$, Simone Aparecida Capellini ${ }^{3}$
}

\begin{abstract}
RESUMO
Objetivo: o objetivo deste estudo foi comparar o desempenho cognitivolinguístico e em leitura de escolares com Transtorno de Déficit de Atenção e Hiperatividade com escolares sem queixa de transtornos comportamentais e/ ou de aprendizagem. Método: participaram deste estudo 20 escolares de $5^{\circ}$ a $8^{\circ}$ ano do ensino fundamental. Os escolares foram divididos em dois: Grupo I (GI): composto por 10 escolares com diagnóstico interdisciplinar de Transtorno de Déficit de Atenção e Hiperatividade, e Grupo II (GII): composto por 10 escolares sem queixa de transtornos comportamentais e/ou de aprendizagem. Como procedimento foi realizado o Teste de habilidades metalinguísticas e leitura (PROHMELE), composto de testes de identificação de sílabas e fonemas, manipulação de sílabas e fonemas, repetição de pseudopalavras e leitura. Resultados: os resultados apresentaram diferença estatisticamente significante entre GI e GII, demonstrando que os escolares do grupo GI apresentaram desempenho superior quando comparados com os escolares do grupo GII. Conclusão: de acordo com os resultados deste estudo podemos concluir que as dificuldades apresentadas por escolares com Transtorno de Déficit de Atenção e Hiperatividade podem ser atribuídas à desatenção, hiperatividade e desorganização, característica do próprio diagnóstico, e não a uma desordem de linguagem de base fonológica.
\end{abstract}

Palavras-chave: aprendizagem; transtorno de déficit de atenção com hiperatividade; leitura

1 Doutoranda do Programa de Pós Graduação em Educação da Faculdade de Filosofia e Ciências da Universidade Estadual Paulista - FFC/UNESP-Marília - SP / Brasil.

2 Doutoranda do Programa de Pós Graduação em Educação da Faculdade de Filosofia e Ciências da Universidade Estadual Paulista - FFC/UNESP-Marília - SP / Brasil.

3 Livre Docente em Linguagem Escrita - Faculdade de Filosofia e Ciências da Universidade Estadual Paulista - FFC/ UNESP-Marília - SP / Brasil. Docente do Departamento de Fonoaudiologia e do Programa de Pós-Graduação em Educação da Faculdade de Filosofia e Ciências da Universidade Estadual Paulista - FFC/UNESP-Marília - SP / Brasil.

Trabalho realizado no Centro de Estudos da Educação e Saúde - CEES/UNESP -Faculdade de Filosofia e Ciências da Universidade Estadual Paulista - Marília SP.

Correspondência para: claudiasilvafono@yahoo.com.br

Apoio: CNPq

Como citar este artigo: Silva C, Cunha VLO, Capellini SA. Performance cognitive-linguistic and reading of students with Attention Deficit and Hyperactivity Disorder. Journal of Human Growth and Development 2011; 21(3): 849-858.2011; 21(3): 841-848.

Artigo submetido em 05.03.11, aceito em 20.09.11. 


\begin{abstract}
ABSRTRACT
Objective: the aim of this study was to compare the cognitive-linguistic skills performance and reading of students with Attention Deficit and Hyperactivity Disorder and students without behavioral and/or learning disorders. Method: the study included 20 students from $5^{\text {th }}$ to $8^{\text {th }}$ grade of elementary school. The students were divided into: Group I (GI): composed by 10 students with an interdisciplinary diagnosis of Attention Deficit and Hyperactivity Disorder, and Group II (GII): composed by 10 students without complaints of behavioral and/ or learning disorders. The tests of metalinguistic skills and reading (PROHMELE) were used as procedure, composed of syllabic and phonemic identification, syllabic and phonemic manipulation, repetition of nonwords and reading tests. Results: the results showed statistically significant differences between GI and GII, demonstrating that students from GI presented superior performances when compared to the students from GII. Conclusion: according to the findings of this study we can conclude that the difficulties presented by students with Attention Deficit and Hyperactivity Disorder can be attributed to inattention, hyperactivity and disorganization, characteristic of this diagnosis, and not to a disorder of language of phonological basis.
\end{abstract}

Key words: learning; attention deficit and hyperactivity disorder; reading

\section{INTRODUÇÃO}

O Transtorno de Déficit de Atenção e Hiperatividade (TDAH) é uma alteração comportamental comum, caracterizada por baixos níveis de atenção e concentração, e por altos níveis de atividade psicomotora, desatenção e impulsivida$\mathrm{de}^{1}$. Havendo comorbidade com problemas de aprendizagem, frequentemente associado a comprometimentos acadêmico, social e profissional. Estudos mostram que cerca de $80 \%$ das crianças com TDAH exibem problemas de aprendizagem e/ou desempenho acadêmico, e que os maiores riscos para essa população são a fraca conquista acadêmica e taxas superiores de abandono à escola ${ }^{2,3}$.

As características principais do TDAH (desatenção, impulsividade e hiperatividade) podem levar a diversas dificuldades no contexto escolar. Pois, muitas vezes, esses escolares apresentam problemas para sustentar a atenção em tarefas que exigem concentração, organização, finalização de trabalhos independentes e o aprendizado de um novo conteúdo ${ }^{2}$.

O diagnóstico do TDAH raramente é realizado antes da fase escolar, mesmo que a sintomatologia do transtorno esteja presente em fases anteriores à pré-escolar, pois nestes casos, a desatenção, a hiperatividade e/ou impulsividade persiste por vários meses, sugerindo o diagnóstico. Em outros casos, os sintomas podem surgir a partir do momento em que a demanda ambiental passa a ser maior; onde as habilidades relacionadas à função executiva, como planejamento, organização e persistência do foco atencional, tornam-se ainda mais imprescindíveis para a realização de tarefas $^{2,4-6}$.

As características de alterações linguísticas, mais comuns, de escolares com TDAH relacionadas ao desempenho acadêmico são: transtornos de organização sequencial e temporal de fonemas na fala e na escrita, dificuldade em regular a intensidade e velocidade do discurso, recursos linguísticos escassos, ausência de organização textual, problemas na leitura para decodificação, podendo apresentar omissões e substituições de palavras e fonemas, ocorrendo o mesmo na escrita com alteração da ordem lógica das orações e produção textual desorganizada. Os aspectos linguísticos mais prejudicados nesses escolares são o fonológico, o sintático e o pragmático, sendo que, as dificuldades para os aspectos fonético-fonológicos e gramaticais são causados, provavelmente, por uma dificuldade de atenção e de controle inibitório dos estímulos irrelevantes, do que 
por uma incapacidade específica para manejar os aspectos linguísticos ${ }^{3,6,7}$.

As dificuldades de atenção e de hiperatividade apresentadas por escolares com TDAH podem comprometer seu desempenho acadêmico, pois o déficit de linguagem apresentado pode interferir na aprendizagem do sistema de escrita alfabético, visto que, habilidades subjacentes a este processo, como as habilidades de consciência fonológica, quando alteradas, prejudicam esta aquisição $0^{8-10}$.

Dessa forma, em situação de sala de aula, os escolares que apresentam patologias que acarretem em prejuízo para o desenvolvimento de suas habilidades acadêmicas, como o TDAH, podem se sentir desmotivados e desinteressados devido às dificuldades cognitivo-linguísticas, que comprometem a compreensão do material lido ou escrito ${ }^{6}$.

Considerando os aspectos expostos acima, este artigo teve como objetivo comparar o desempenho cognitivolinguístico e em leitura de escolares com TDAH e escolares sem queixa de transtornos comportamentais e/ou de aprendizagem.

\section{MÉTODO}

Este trabalho foi aprovado pelo Comitê de Ética em Pesquisa da Faculdade de Filosofia e Ciências da Universidade Estadual Paulista - FFC/UNESP/ Marília - SP, sob o protocolo de número 3326/2006.

Participaram deste estudo 20 escolares do $5^{\circ}$ ao $8^{\circ}$ ano do ensino fundamental de uma escola municipal, sendo 14 escolares do gênero masculino e 6 do gênero feminino, na faixa etária de 9 a 13 anos. Os escolares deste estudo foram divididos em dois grupos:

- Grupo I (GI): composto por 10 escolares com diagnóstico interdisciplinar de TDAH.

O diagnóstico de TDAH desses escolares foi realizado por equipe interdisciplinar do Laboratório de Investigação dos Desvios da Aprendizagem do Centro de Estudos da Educação e Saúde - CEES/ UNESP - Marília, incluindo avaliação fo- noaudiológica, neurológica, neuropsicológica e seguindo critérios propostos pelo DSM-IV ${ }^{11}$. Todos os escolares deste grupo faziam uso de medicação por, pelo menos, seis meses.

- Grupo II (GII): composto por 10 escolares sem queixa de transtornos comportamentais e/ou de aprendizagem. Os escolares do grupo GII foram pareados com os escolares do GI de acordo com a idade e série escolar.

Esses escolares foram indicados por seus professores seguindo o critério de desempenho acadêmico satisfatório em dois bimestres consecutivos (média igual ou superior a 7). A partir dessa indicação, os escolares foram submetidos previamente às avaliações otorrinolaringológica, audiológica e oftalmológica, e somente participaram deste estudo aqueles que apresentaram resultados dentro dos padrões de normalidade. A classificação do nível socioeconômico foi realizada com base no estudo estatístico do Índice de Desenvolvimento Socioeconômico $(\text { IDESE })^{12}$, garantindo, assim, a homogeneidade da amostra do ponto de vista socioeconômico.

Como critério de inclusão, foi utilizado o Termo de Consentimento Livre e Esclarecido assinado, ausência de queixa auditiva ou visual descritas nos prontuários dos escolares do GII e o diagnóstico interdisciplinar de TDAH para os escolares do GII.

Como critério de exclusão, foi considerado a não assinatura dos pais ou responsáveis do Termo de Consentimento Livre e Esclarecido, a presença de deficiência sensorial, motora ou cognitiva referida em prontuário escolar.

Anteriormente ao início da aplicação do procedimento, os pais ou responsáveis pelos escolares selecionados assinaram o termo de Consentimento Livre e Esclarecido autorizando a realização do estudo, conforme resolução do Conselho Nacional de Saúde CNS 196/96.

Como procedimento foram utilizadas as Provas de Habilidades Metalinguísticas e de Leitura do PROHMELE ${ }^{13}$. As provas aplicadas encontram-se descritas a seguir: 
Provas de identificação silábica e fonêmica: Identificação de sílaba inicial (ISI), Identificação de fonema inicial (IFI), Identificação de sílaba final (ISF), Identificação de fonema final (IFF), Identificação de sílaba medial (ISM), Identificação de fonema medial (IFM);

Provas de manipulação silábica e fonêmica: Segmentação silábica (Seg Sil), Segmentação fonêmica (Seg Fon), Adição silábica (Ad Sil), Adição fonêmica (Ad Fon), Substituição silábica (Subs Sil), Substituição fonêmica (Subs Fon), Subtração silábica (Subt Sil), Subtração fonêmica (Subt Fon), Combinação silábica (Com Sil), Combinação fonêmica (Com Fon);

Repetição de Não-Palavras: Repetição de Não-Palavras monossilábicas (RNP M1; RNP M2); Repetição de NãoPalavras dissilábicas (RNP D1; RNP D2): Repetição de Não-Palavras trissilábicas (RNP T1; RNP T2); Repetição de NãoPalavras polissilábicas com 4 sílabas (RNP P4-1; RNP P4-2); Repetição de Não-Palavras polissilábicas com 5 sílabas (RNP P5-1; RNP P5-2); Repetição de Não-Palavras polissilábicas com 6 sílabas (RNP P6-1; RNP P6-2);

Provas de Leitura: Leitura de palavras reais onde foi apresentada uma lista de palavras reais isoladas (133 palavras); e Leitura de pseudopalavras onde foi apresentada uma lista de pseudopalavras (27 pseudopalavras).

As pseudopalavras são entendidas aqui, como logatomo, ou seja, uma sílaba ou uma seqüência de sílabas que pertencem à língua, mas que não formam uma palavra com significado ${ }^{14}$.

A aplicação das provas de habilidades metalinguísticas foi realizada de forma que o escolar não tivesse pista visual da articulação dos sons produzidos pela examinadora. As respostas do escolar foram anotadas na folha de respostas do PROHMELE. O escolar foi instruído e treinado previamente, por meio de exemplos similares aos da prova, para que soubesse o que deveria fazer.

As provas de leitura foram realizadas em voz alta e gravadas em gravador digital para posterior análise da leitura. Cada escolar recebeu instrução de como deveria ler as listas de palavras, apresentadas no formato de letra Arial tamanho 14, espaço duplo, divididas em colunas segundo extensão de palavras (monossilábicas, dissilábicas, trissilábicas e polissilábicas - 4 a 7 sílabas) e de pseudopalavras (monossilábicas, dissilábicas, trissilábicas). Na prova de leitura de pseudopalavras, foi esclarecido aos escolares que os mesmos iriam ler palavras que não existem, e que por isto não faz parte de seu vocabulário.

O número de palavras que compõem a prova de leitura de palavras reais e pseudopalavras é diferente, pelo fato de que, a primeira lista foi composta de palavras segundo as regras de correspondência grafofonêmica independente do contexto e de correspondência grafofonêmica dependente do contexto, enquanto que a segunda lista apenas foi composta de pseudopalavras, derivadas de palavras segundo a regra de correspondência grafofonêmica independente do contexto. Todas as provas deste estudo foram analisadas segundo o critério de erros.

A caracterização dos tipos de erros da leitura de palavras reais e pseudopalavras foi realizada a partir de critérios estabelecidos para o Português do Brasil ${ }^{14}$, descritos a seguir:

D1 - Regra de correspondência grafofonêmica independente do contexto referentes às palavras regulares com correspondência unívoca.

D2 - Regra de correspondência grafofonêmica dependente do contexto referentes às regras aplicadas às palavras irregulares.

D4- Valores da letra " $X$ " dependentes exclusivamente do léxico mental e ortográfico.

$\mathrm{Na}$ prova de leitura de pseudopalavras será considerada somente a regra $D 1$, pois seu objetivo é verificar a correspondência unívoca entre letra e som.

Os escolares do GI foram avaliados após 30 minutos da administração do medicamento (metilfenidato), uma vez que na ausência da medicação não foi possível realizar a avaliação proposta 
neste estudo. Foram realizadas duas sessões individuais para a avaliação, com duração de 30 minutos cada. Os escolares do GII foram avaliados, também individualmente, em uma sala de aula fornecida pela coordenação pedagógica, em horário pré-determinado pelo professor de cada escolar.

Os resultados foram analisados estatisticamente com nível de significância de $5 \%(0,050)$ para a aplicação dos testes estatísticos, utilizando o programa SPSS (Statistical Package for Social Sciences), em sua versão 19.0. O teste utilizado para análise estatística foi o
Teste de Mann-Whitney. Os resultados estatisticamente significantes foram marcados com um asterisco $(*)$.

\section{RESULTADOS}

Quando aplicado o Teste de MannWhitney, com o intuito de verificar possíveis diferenças entre os grupos estudados para as variáveis de interesse, foi possível observar que, de acordo com a Tabela 1, não houve diferença estatisticamente significante para as provas de identificação de sílabas e fonemas.

Tabela 1: Distribuição da média, desvio-padrão, valores de referência mínima e máxima, e valor de p na comparação do desempenho entre GI e GII nas provas de identificação de sílabas e fonemas

\begin{tabular}{ccccccc}
\hline Variáveis & Grupo & Média & Desvio-padrão & Mínimo & Máximo & Valor de p \\
\hline \multirow{2}{*}{ ISI } & I & 0,40 & 1,27 & 0,00 & 4,00 & 0,317 \\
& II & 0,00 & 0,00 & 0,00 & 0,00 & \\
\multirow{2}{*}{ IFI } & I & 0,200 & 0,42 & 0,00 & 1,00 & 0,626 \\
& II & 0,20 & 0,63 & 0,00 & 2,00 & \\
\multirow{2}{*}{ IFI } & I & 0,10 & 0,32 & 0,00 & 1,00 & 0,503 \\
& II & 0,40 & 0,97 & 0,00 & 3,00 & \\
IFF & I & 1,00 & 1,89 & 0,00 & 5,00 & 0,234 \\
\multirow{2}{*}{ ISM } & II & 0,10 & 0,32 & 0,00 & 1,00 & \\
& I & 0,60 & 1,27 & 0,00 & 4,00 & 0,068 \\
ISM & II & 0,00 & 0,00 & 0,00 & 0,00 & \\
& I & 1,40 & 1,84 & 0,00 & 5,00 & 0,325 \\
\hline
\end{tabular}

Legenda: ISI: identificação de sílaba inicial, IFI: identificação de fonema inicial, ISF: identificação de sílaba final, IFF: identificação de fonema final, ISM: identificação de sílaba medial, IFM: identificação de fonema medial

A Tabela 2 apresenta os dados da comparação dos grupos GI e GII nas provas de manipulação de sílabas e fonemas, sendo possível observar que houve diferença estatisticamente significante para as provas de Segmentação fonêmica, Adição silábica, Adição fonêmica, Substituição silábica, Substituição fonêmica, Combinação silábica e Combinação fonêmica, indicando que o grupo GII apresentou desempenho superior quando comparado ao grupo GI.

Nas provas de leitura de palavras reais e de pseudopalavras houve diferença estatisticamente significante para as palavras referentes à regra D1, que são as palavras regulares, com correspondência grafofonêmica independente do contexto, de acordo com os dados da tabela 3.

De acordo com a tabela 4, houve diferença estatisticamente significante entre os grupos GI e GII somente para as regras D2.3 e D2.5, na comparação dos resultados da leitura de palavras reais, para a regra D2.1 a D2.11, correspondente às palavras irregulares, com correspondência grafofonêmica depen- 
Tabela 2: Distribuição da média, desvio-padrão, valores de referência mínima e máxima, e valor de p na comparação do desempenho entre GI e GII nas provas de manipulação de sílabas e fonemas

\begin{tabular}{|c|c|c|c|c|c|c|}
\hline Variáveis & Grupo & Média & Desvio-padrão & Mínimo & Máximo & Valor de p \\
\hline Seg Sil & $\begin{array}{c}\text { I } \\
\text { II }\end{array}$ & $\begin{array}{l}0,30 \\
0,30\end{array}$ & $\begin{array}{l}0,48 \\
0,48\end{array}$ & $\begin{array}{l}0,00 \\
0,00\end{array}$ & $\begin{array}{l}1,00 \\
1,00\end{array}$ & $>0,999$ \\
\hline Seg Fon & $\begin{array}{c}\text { I } \\
\text { II }\end{array}$ & $\begin{array}{l}7,60 \\
1,50\end{array}$ & $\begin{array}{l}3,98 \\
1,27\end{array}$ & $\begin{array}{l}0,00 \\
0,00\end{array}$ & $\begin{array}{c}10,00 \\
4,00\end{array}$ & $0,004 *$ \\
\hline Ad Sil & $\begin{array}{c}\text { I } \\
\text { II }\end{array}$ & $\begin{array}{l}2,10 \\
0,10\end{array}$ & $\begin{array}{l}3,18 \\
0,32\end{array}$ & $\begin{array}{l}0,00 \\
0,00\end{array}$ & $\begin{array}{c}10,00 \\
1,00\end{array}$ & $0,040 *$ \\
\hline Ad Fon & $\begin{array}{c}\text { I } \\
\text { II }\end{array}$ & $\begin{array}{l}7,30 \\
0,00\end{array}$ & $\begin{array}{l}3,89 \\
0,00\end{array}$ & $\begin{array}{l}0,00 \\
0,00\end{array}$ & $\begin{array}{c}10,00 \\
0,00\end{array}$ & $<0,001 *$ \\
\hline Subs Sil & $\begin{array}{c}\text { I } \\
\text { II }\end{array}$ & $\begin{array}{l}4,70 \\
0,10\end{array}$ & $\begin{array}{l}4,72 \\
0,32\end{array}$ & $\begin{array}{l}0,00 \\
0,00\end{array}$ & $\begin{array}{c}10,00 \\
1,00\end{array}$ & $0,005^{*}$ \\
\hline Subs Fon & $\begin{array}{c}\text { I } \\
\text { II }\end{array}$ & $\begin{array}{l}6,20 \\
0,30\end{array}$ & $\begin{array}{l}4,92 \\
0,68\end{array}$ & $\begin{array}{l}0,00 \\
0,00\end{array}$ & $\begin{array}{c}10,00 \\
2,00\end{array}$ & $0,004 *$ \\
\hline Subt Sil & $\begin{array}{c}\text { I } \\
\text { II }\end{array}$ & $\begin{array}{l}1,60 \\
0,60\end{array}$ & $\begin{array}{l}1,78 \\
1,08\end{array}$ & $\begin{array}{l}0,00 \\
0,00\end{array}$ & $\begin{array}{l}4,00 \\
3,00\end{array}$ & 0,199 \\
\hline Subt Fon & $\begin{array}{c}\text { I } \\
\text { II }\end{array}$ & $\begin{array}{l}4,90 \\
0,80\end{array}$ & $\begin{array}{l}4,86 \\
1,14\end{array}$ & $\begin{array}{l}0,00 \\
0,00\end{array}$ & $\begin{array}{c}10,00 \\
3,00\end{array}$ & 0,104 \\
\hline Com Sil & $\begin{array}{c}\text { I } \\
\text { II }\end{array}$ & $\begin{array}{l}2,20 \\
0,50\end{array}$ & $\begin{array}{l}1,87 \\
1,27\end{array}$ & $\begin{array}{l}0,00 \\
0,00\end{array}$ & $\begin{array}{l}5,00 \\
4,00\end{array}$ & $0,028 *$ \\
\hline Com Fom & $\begin{array}{c}\text { I } \\
\text { II }\end{array}$ & $\begin{array}{l}7,30 \\
1,20\end{array}$ & $\begin{array}{l}4,42 \\
1,23\end{array}$ & $\begin{array}{l}0,00 \\
0,00\end{array}$ & $\begin{array}{c}10,00 \\
4,00\end{array}$ & $0,012 *$ \\
\hline
\end{tabular}

Legenda: Seg Sil: segmentação silábica, Seg Fon: segmentação fonêmica, Ad Sil: adição silábica, Ad Fon: adição fonêmica, Subs Sil: substituição silábica, Subs Fon: substituição fonêmica, Subt Sil: subtração silábica, Subt Fon: subtração fonemica, Com Sil: combinação de sílabas, Com Fon: Combinação de fonemas

Tabela 3: Distribuição da média, desvio-padrão, valores de referência mínima e máxima, e valor de p na comparação do desempenho entre os grupos GI e GII para as regras D1 na leitura de palavras reais e pseudopalavras

\begin{tabular}{ccccccc}
\hline Variáveis & Grupo & Média & Desvio-padrão & Mínimo & Máximo & Valor de p \\
\hline \multirow{2}{*}{ D1 PR } & I & 8,80 & 14,18 & 0,00 & 45,00 & \multirow{2}{*}{$0,024^{*}$} \\
& II & 0,44 & 0,88 & 0,00 & 2,00 & \\
\multirow{2}{*}{ D1 PP } & I & 3,80 & 4,19 & 0,00 & 14,00 & $0,007^{*}$ \\
& II & 0,40 & 0,52 & 0,00 & 1,00 & \\
\hline
\end{tabular}

Legenda: D1 PR: regra de correspondência grafofonêmica independente do contexto para palavras reais; D1 PP: regra de correspondência grafofonêmica independente do contexto para pseudopalavras

dente do contexto. Sendo que o grupo GII apresentou desempenho superior em relação ao grupo GI. Lembrando que, a regra D2.3 se refere a leitura do grafema
" $\mathrm{s}$ " em final de sílaba interna; e a regra D2.5 se refere a leitura do grafema " $z$ " em início de vocábulo e em início de sílaba. 
Tabela 4: Distribuição da média, desvio-padrão, valores de referência mínima e máxima, e valor de p na comparação do desempenho entre os grupos GI e GII para as regras D2.1 a D2.11 na leitura de palavras reais

\begin{tabular}{|c|c|c|c|c|c|c|}
\hline Variáveis & Grupo & Média & Desvio-padrão & Mínimo & Máximo & Valor de $p$ \\
\hline $\mathrm{D} 2.1 \mathrm{PR}$ & $\begin{array}{c}\text { I } \\
\text { II }\end{array}$ & $\begin{array}{l}0,44 \\
0,33\end{array}$ & $\begin{array}{l}1,01 \\
1,00\end{array}$ & $\begin{array}{l}0,00 \\
0,00\end{array}$ & $\begin{array}{l}3,00 \\
3,00\end{array}$ & 0,586 \\
\hline D 2.2 & $\begin{array}{c}\text { I } \\
\text { II }\end{array}$ & $\begin{array}{l}3,30 \\
0,22\end{array}$ & $\begin{array}{l}7,38 \\
0,44\end{array}$ & $\begin{array}{l}0,00 \\
0,00\end{array}$ & $\begin{array}{c}24,00 \\
1,00\end{array}$ & 0,118 \\
\hline D 2.3 & $\begin{array}{c}\text { I } \\
\text { II }\end{array}$ & $\begin{array}{l}1,30 \\
0,00\end{array}$ & $\begin{array}{l}2,11 \\
0,00\end{array}$ & $\begin{array}{l}0,00 \\
0,00\end{array}$ & $\begin{array}{l}6,00 \\
0,00\end{array}$ & $0,039 *$ \\
\hline D 2.4 & $\begin{array}{l}\text { I } \\
\text { II }\end{array}$ & $\begin{array}{l}0,22 \\
0,22\end{array}$ & $\begin{array}{l}0,44 \\
0,67\end{array}$ & $\begin{array}{l}0,00 \\
0,00\end{array}$ & $\begin{array}{l}1,00 \\
2,00\end{array}$ & 0,634 \\
\hline D 2.5 & $\begin{array}{c}\text { I } \\
\text { II }\end{array}$ & $\begin{array}{l}0,70 \\
0,00\end{array}$ & $\begin{array}{l}0,95 \\
0,00\end{array}$ & $\begin{array}{l}0,00 \\
0,00\end{array}$ & $\begin{array}{l}2,00 \\
0,00\end{array}$ & $0,039 *$ \\
\hline D 2.6.1 & $\begin{array}{l}\text { I } \\
\text { II }\end{array}$ & $\begin{array}{l}0,60 \\
0,22\end{array}$ & $\begin{array}{l}1,08 \\
0,67\end{array}$ & $\begin{array}{l}0,00 \\
0,00\end{array}$ & $\begin{array}{l}3,00 \\
2,00\end{array}$ & 0,330 \\
\hline D 2.6.3 & $\begin{array}{l}\text { I } \\
\text { II }\end{array}$ & $\begin{array}{l}1,20 \\
0,78\end{array}$ & $\begin{array}{l}2,10 \\
1,39\end{array}$ & $\begin{array}{l}0,00 \\
0,00\end{array}$ & $\begin{array}{l}6,00 \\
4,00\end{array}$ & 0,741 \\
\hline D 2.6.4 & $\begin{array}{l}\text { I } \\
\text { II }\end{array}$ & $\begin{array}{l}0,60 \\
0,63\end{array}$ & $\begin{array}{l}0,52 \\
0,92\end{array}$ & $\begin{array}{l}0,00 \\
0,00\end{array}$ & $\begin{array}{l}1,00 \\
2,00\end{array}$ & 0,768 \\
\hline D 2.6 .5 & $\begin{array}{l}\text { I } \\
\text { II }\end{array}$ & $\begin{array}{l}0,00 \\
0,00\end{array}$ & $\begin{array}{l}0,00 \\
0,00\end{array}$ & $\begin{array}{l}0,00 \\
0,00\end{array}$ & $\begin{array}{l}0,00 \\
0,00\end{array}$ & $>0,999$ \\
\hline D 2.7 & $\begin{array}{l}\text { I } \\
\text { II }\end{array}$ & $\begin{array}{l}1,30 \\
0,00\end{array}$ & $\begin{array}{l}3,13 \\
0,00\end{array}$ & $\begin{array}{l}0,00 \\
0,00\end{array}$ & $\begin{array}{c}10,00 \\
0,00\end{array}$ & 0,068 \\
\hline D 2.8 & $\begin{array}{l}\text { I } \\
\text { II }\end{array}$ & $\begin{array}{l}0,50 \\
0,20\end{array}$ & $\begin{array}{l}0,71 \\
0,63\end{array}$ & $\begin{array}{l}0,00 \\
0,00\end{array}$ & $\begin{array}{l}2,00 \\
2,00\end{array}$ & 0,178 \\
\hline D 2.9 & $\begin{array}{l}\text { I } \\
\text { II }\end{array}$ & $\begin{array}{l}0,50 \\
0,00\end{array}$ & $\begin{array}{l}1,07 \\
0,00\end{array}$ & $\begin{array}{l}0,00 \\
0,00\end{array}$ & $\begin{array}{l}3,00 \\
0,00\end{array}$ & 0,104 \\
\hline D 2.10 & $\begin{array}{c}\text { I } \\
\text { II }\end{array}$ & $\begin{array}{l}0,50 \\
0,00\end{array}$ & $\begin{array}{l}1,41 \\
0,00\end{array}$ & $\begin{array}{l}0,00 \\
0,00\end{array}$ & $\begin{array}{l}4,00 \\
0,00\end{array}$ & 0,264 \\
\hline D 2.11 & $\begin{array}{c}\text { I } \\
\text { II }\end{array}$ & $\begin{array}{l}1,56 \\
0,00\end{array}$ & $\begin{array}{l}4,30 \\
0,00\end{array}$ & $\begin{array}{l}0,00 \\
0,00\end{array}$ & $\begin{array}{c}13,00 \\
0,00\end{array}$ & 0,126 \\
\hline
\end{tabular}

Legenda: D2: Regra de correspondência grafofonêmica dependente do contexto para palavras reais

A tabela 5 apresenta os resultados da leitura de palavras reais para a regra D2.12 a D2.23, correspondente às palavras irregulares, com correspondência grafofonêmica dependente do contexto, onde foi possível observar que houve diferença estatisticamente significante entre os grupos somente para a regra D2.23, que se refere a regra correspondente à leitura dos grafemas "i" e " $u$ ". Sendo que o grupo GII apresentou desempenho superior em relação ao grupo GI.
Na tabela 6 foi possível observar que houve diferença estatisticamente significante entre os grupos, com desempenho superior para o grupo GII na comparação dos resultados da leitura de palavras reais para a regra D4, sendo esta regra referente a três valores atribuídos à letra " $x$ ", que dependem exclusivamente da internalização do léxico mental ortográfico e de suas relações com o léxico mental fonológico. 
Tabela 5: Distribuição da média, desvio-padrão, valores de referência mínima e máxima, e valor de p na comparação do desempenho entre os grupos GI e GII para as regras D2.12 a D2.23 na leitura de palavras reais

\begin{tabular}{|c|c|c|c|c|c|c|}
\hline Variáveis & Grupo & Média & Desvio-padrão & Mínimo & Máximo & Valor de $p$ \\
\hline D 2.12 & $\begin{array}{c}\text { I } \\
\text { II }\end{array}$ & $\begin{array}{l}0,25 \\
0,20\end{array}$ & $\begin{array}{l}0,71 \\
0,63\end{array}$ & $\begin{array}{l}0,00 \\
0,00\end{array}$ & $\begin{array}{l}2,00 \\
2,00\end{array}$ & 0,871 \\
\hline D 2.13 & $\begin{array}{l}\text { I } \\
\text { II }\end{array}$ & $\begin{array}{l}2,00 \\
0,10\end{array}$ & $\begin{array}{l}4,40 \\
0,32\end{array}$ & $\begin{array}{l}0,00 \\
0,00\end{array}$ & $\begin{array}{c}14,00 \\
1,00\end{array}$ & 0,213 \\
\hline D 2.14 & $\begin{array}{l}\text { I } \\
\text { II }\end{array}$ & $\begin{array}{l}0,11 \\
0,30\end{array}$ & $\begin{array}{l}0,33 \\
0,95\end{array}$ & $\begin{array}{l}0,00 \\
0,00\end{array}$ & $\begin{array}{l}1,00 \\
3,00\end{array}$ & $>0,999$ \\
\hline D 2.15 & $\begin{array}{l}\text { I } \\
\text { II }\end{array}$ & $\begin{array}{l}2,20 \\
0,10\end{array}$ & $\begin{array}{l}5,94 \\
0,32\end{array}$ & $\begin{array}{l}0,00 \\
0,00\end{array}$ & $\begin{array}{c}19,00 \\
1,00\end{array}$ & 0,234 \\
\hline D 2.16 & $\begin{array}{l}\text { I } \\
\text { II }\end{array}$ & $\begin{array}{l}0,33 \\
0,30\end{array}$ & $\begin{array}{l}0,71 \\
0,95\end{array}$ & $\begin{array}{l}0,00 \\
0,00\end{array}$ & $\begin{array}{c}2,00 \\
3,0\end{array}$ & 0,563 \\
\hline D 2.17 & $\begin{array}{l}\text { I } \\
\text { II }\end{array}$ & $\begin{array}{l}3,60 \\
0,10\end{array}$ & $\begin{array}{l}8,70 \\
0,32\end{array}$ & $\begin{array}{l}0,00 \\
0,00\end{array}$ & $\begin{array}{c}28,00 \\
1,00\end{array}$ & 0,101 \\
\hline D 2.18 & $\begin{array}{l}\text { I } \\
\text { II }\end{array}$ & $\begin{array}{l}0,25 \\
0,22\end{array}$ & $\begin{array}{l}0,71 \\
0,44\end{array}$ & $\begin{array}{l}0,00 \\
0,00\end{array}$ & $\begin{array}{l}2,00 \\
1,00\end{array}$ & 0,717 \\
\hline D 2.19 .1 & $\begin{array}{c}\text { I } \\
\text { II }\end{array}$ & $\begin{array}{l}0,30 \\
0,40\end{array}$ & $\begin{array}{l}0,68 \\
0,84\end{array}$ & $\begin{array}{l}0,00 \\
0,00\end{array}$ & $\begin{array}{l}2,00 \\
2,00\end{array}$ & 0,914 \\
\hline D 2.19 .2 & $\begin{array}{c}\text { I } \\
\text { II }\end{array}$ & $\begin{array}{l}1,00 \\
1,00\end{array}$ & $\begin{array}{l}2,65 \\
1,49\end{array}$ & $\begin{array}{l}0,00 \\
0,00\end{array}$ & $\begin{array}{l}8,00 \\
4,00\end{array}$ & 0,458 \\
\hline $\mathrm{D} 2.21$ & $\begin{array}{c}\text { I } \\
\text { II }\end{array}$ & $\begin{array}{l}0,70 \\
0,30\end{array}$ & $\begin{array}{l}1,57 \\
0,68\end{array}$ & $\begin{array}{l}0,00 \\
0,00\end{array}$ & $\begin{array}{l}5,00 \\
2,00\end{array}$ & 0,618 \\
\hline D 2.22 & $\begin{array}{c}\text { I } \\
\text { II }\end{array}$ & $\begin{array}{l}3,60 \\
0,20\end{array}$ & $\begin{array}{c}10,01 \\
0,63\end{array}$ & $\begin{array}{l}0,00 \\
0,00\end{array}$ & $\begin{array}{c}32,00 \\
2,00\end{array}$ & 0,255 \\
\hline D 2.23 & $\begin{array}{c}\text { I } \\
\text { II }\end{array}$ & $\begin{array}{l}3,20 \\
0,10\end{array}$ & $\begin{array}{l}8,39 \\
0,32\end{array}$ & $\begin{array}{l}0,00 \\
0,00\end{array}$ & $\begin{array}{c}27,00 \\
1,00\end{array}$ & $0,049 *$ \\
\hline
\end{tabular}

Legenda: D2: Regra de correspondência grafofonêmica dependente do contexto para palavras reais

Tabela 6: Distribuição da média, desvio-padrão, valores de referência mínima e máxima, e valor de p na comparação do desempenho entre os grupos GI e GII para as regras D4 na leitura de palavras reais

\begin{tabular}{ccccccc}
\hline Variável & Grupo & Média & Desvio-padrão & Mínimo & Máximo Valor de $\mathbf{p}$ \\
\hline \multirow{2}{*}{ D4 } & I & 1,50 & 1,51 & 0,00 & 4,00 & \multirow{2}{*}{$0,045^{*}$} \\
& II & 0,22 & 0,44 & 0,00 & 1,00 & \\
\hline
\end{tabular}

Legenda: D4: valores da letra " $X$ " dependentes exclusivamente do léxico mental e ortográfico

\section{DISCUSSÃO}

Nossos resultados revelaram que, os escolares do grupo GII obtiveram desempenho superior aos escolares do grupo GI, ocorrendo diferença estatisticamente significante nas provas de manipulação silábica e fonêmica, para a segmentação fonêmica, adição, substituição e combinação silábica e fonêmica. Sendo que essas provas necessitam, para sua realização, de maior concentração e atenção para a identificação das sílabas e fonemas trabalhados.

Estudos recentes $6,10,13,15$ relataram que algumas tarefas, como as de mani- 
pulação de sílabas e fonemas, são mais complexas, pois exigem a realização de duas operações (armazenar uma unidade na memória enquanto é realizada uma nova operação), e tarefas de identificação de sílabas e fonemas são consideradas mais simples (exigem apenas uma operação seguida de resposta); dessa forma, o desempenho em tarefas que verificam as habilidades metalinguísticas, pode variar de acordo com o tipo de operação que é solicitada à criança.

Estudos investigaram o componente semântico de escolares com TDAH comparados com escolares sem dificuldades de aprendizagem e/ou comportamental, por meio de tarefas de organização semântica, memória auditiva e fluência verbal, e constataram valores próximos aos dos sujeitos normais. Dessa forma, concluíram que as dificuldades para os aspectos fonético-fonológicos e gramaticais, encontradas nos escolares com TDAH, são causadas, possivelmente, por uma dificuldade de atenção e de controle inibitório dos estímulos irrelevantes do que por uma incapacidade específica para manejar os aspectos linguísticos ${ }^{8,9}$.

Os resultados encontrados no desempenho dos escolares com TDAH para as habilidades metalinguísticas podem ter sofrido interferência de fatores característicos do próprio diagnóstico, uma vez que a desatenção e a hiperatividade podem prejudicar a retenção da informação, interferindo diretamente no processamento auditivo e visual desses escolares $^{8-10}$.

Os sintomas da alteração de processamento auditivo coincidem com os sintomas de TDAH, porém, os sintomas de processamento auditivo atribuídos aos escolares com TDAH, como dificuldade em seguir instruções, sustentar a atenção e para captar informações auditivas, são alterações apontadas como um fenômeno secundário à desatenção e não como um déficit primário do processamento auditivo ${ }^{15}$.

Apesar de o déficit de atenção geralmente caracterizar as duas alterações, realmente há diferenças em relação à natureza da desatenção observada nas duas alterações, ou seja, o déficit de atenção na criança com TDAH é geralmente persistente e supramodal, enquanto que a criança com alteração de processamento auditivo apresenta uma alteração restrita na atenção auditiva ${ }^{16}$.

Nas provas de leitura de palavras reais e pseudopalavras que envolvem regras houve diferença estatisticamente significante para as regras D1, D2.3, D2.5, D2.23 e D4.

Para que ocorra a decodificação de palavras e pseudopalavras é necessário o envolvimento de algumas habilidades, como o processamento visual e auditivo, - mecanismo de conversão grafema/ fonema, processos atencionais, acesso ao léxico e a memória fonológica. Os escolares com TDAH apresentam alteração nas áreas envolvidas com a demanda atencional, de auto-regulação, memória de trabalho e consciência fonológica, devendo-se a isso, a forte associação encontrada entre a incapacidade de leitura e o TDAH, como observado nos resultados encontrados no presente estudo. No entanto, estudos sugerem que tal dificuldade não é consequência de uma desordem de linguagem de base fonológica, mas sim uma consequência secundária dos problemas de autoregulação e de atenção inerentes ao TDAH $^{6,10}$.

As alterações de leitura encontradas no TDAH são decorrentes de uma desorganização sequencial e temporal dos fonemas necessários para a execução da atividade proposta, pois, a dificuldade no controle da seleção do segmento utilizado, no caso o fonema, associado com a alteração atencional, leva à omissão de fonemas, sílabas e/ou palavras, resultando em uma leitura comprometida ${ }^{17}$.

\section{CONCLUSÃO}

De acordo com os dados obtidos neste estudo podemos concluir que os escolares com TDAH apresentam alteração no seu desempenho em atividades consideradas mais complexas, como a manipulação silábica e fonêmica, não 
apresentando alteração de desempenho em habilidades mais simples, como na identificação de sílabas e fonemas, quando comparado seu desempenho com escolares sem queixa de dificuldades de aprendizagem e/o comportamental. In-

\section{REFERÊNCIAS}

1. American Psychiatric Association. Diagnostic and statistical manual of mental dosorders (DSM-IV) (4th Ed.). Washington, DC: American Psychiatric Association; 2000.

2. DuPaul GJ, Stoner G. TDAH nas escolas. Estratégias de Avaliação e Intervenção. São Paulo: Editora M. Books do Brasil; 2007.

3. Liotti M, Pliszka SR, Higgins $K$, Prez III $R$, Semrud-Clikeman M. Evidence for specificity of ERP abnormalities during response inhibition in ADHD children: A comparison with reading disorder children without ADHD. Brain and Cognition. 2010; (72):228-237.

4. Zorzi JL, Ciasca SM. Análise de erros ortográficos em diferentes problemas de aprendizagem. Revista CEFAC. 2009; 11(3): 406-416.

5. Mangina CA, Beuzeron-Mangina $\mathrm{H}$. Similarities and differences between learning abilities, "Pure" learning disabilities, "Pure" ADHD and comorbid ADHD with learning disabilities. International Journal of Psychophysiology. 2009; (73):170-177.

6. Pinheiro $F H$, Lourenceti MD, Santos LCA. Transtornos do déficit de atenção e hiperatividade: critérios diagnósticos. In: Capellini SA, Germano GD, Cunha VLO, editors. Transtornos de aprendizagem e transtornos da atenção (da Avaliação à Intervenção). São José dos Campos: Editora Pulso; 2010. p. 21-33.

7. van De Voorde S, Roeyers H, Wiersema JR. Error monitoring in children with ADHD or reading disorder: An event-related. Biological Psychology. 2010; (85):176-185.

8. Ygual-Fernández A, Miranda-Casas A, Cervera-Mérida JF. Dificultades en las dimensiones de forma y contenido del lenguaje en los niños con trastorno por déficit de atención con hiperacti- dicando que, as dificuldades apresentadas por escolares com TDAH podem ser atribuídas à desatenção, hiperatividade e desorganização, característica do próprio diagnóstico e não a uma desordem de linguagem de base fonológica.

vidad. Revista de Neurologia Clinica. 2000; (1):193-202.

9. Mulas F, Etchepareborda MC, AbadMas L, Díaz-Lucero A. Transtornos neuropsicológicos de los adolescentes afectos de trastorno por déficit de atención con hiperactividad. Revista de Neurologia. 2006; 43(1):71-81.

10. Asberg J, Dahlgren S, Sandberg AD. Basic reading skills in high-functioning Swedish children with autism spectrum disorders or attention disorder. Research in Autism Spectrum Disorders. 2008; (2):95-109.

11. DSM-IVTM. Manual Diagnóstico e Estatístico de Transtornos Mentais. Porto Alegre: Artes Médicas; 2000.

12. Índice de Desenvolvimento Socioeconômico (IDESE). Fundação de Economia e Estatística [homepage na internet]. Porto Alegre: 2003. [acesso em ago 2010]. Disponível: hhp:// www.fee.tche.br/sitefee/PT/content/ estatísticas/pg_idese.php

13. Cunha VLO, Capellini SA. PROHMELE - Protocolo de habilidades metalingüísticas e de leitura. Rio de Janeiro: Revinter; 2009.

14. Scliar-Cabral L. Princípios do sistema alfabético do português do Brasil. São Paulo: Contexto; 2003.

15. Abdo AGR, Murphy CFB, Schochat E. Habilidades auditivas em crianças com dislexia e transtorno do déficit de atenção e hiperatividade. PróFono Revista de Atualização Científica. 2010; 22(1): 25-30.

16. Schochat E, Scheuer CI, Andrade ER. Attention deficit hyperactivity disorder. In: Larimer, M. Attention deficit hyperactivity disorder-Research Developments. Nova Iorque: Nova Science Publishers, Inc, ; 2005. p.31-54.

17. Silva C, Capellini SA. Eficácia do programa de remediação fonológica e leitura no distúrbio de aprendizagem. Pró-Fono Revista de Atualização Científica. 2010; 22(2):131-138. 